\title{
Anti-inflammatory effect of an eye drops solution based on liposomal ozonated oil in different corneal and anterior segment human diseases
}

\author{
Andrea Attilio Basile, ${ }^{1}$ Magda Cendali, ${ }^{1}$ Giulia Mandelli, ${ }^{1}$ Gaetano Fioretto ${ }^{2}$ \\ ${ }^{1}$ Sunstone Science, Milan; ${ }^{2}$ Fioretto Ophthalmic Center, San Giuseppe Vesuviano (NA), Italy
}

\begin{abstract}
The gaseous ozone can be effectively stabilized - for topical use - as ozonated oil, an organic analog of ozone, that has the same properties; the tolerability on the ocular surface has been further improved by means a specific formulation, developed for ophthalmic use, based on liposomal sunflower ozonated oil plus hypromellose (Ozodrop ${ }^{\circledR}$, FB Vision, Ascoli Piceno, Italy), which is extremely biocompatible with the delicate ocular surface tissue. The use of ozone in certain eye anterior segment pathologies could be providential due to its anti-inflammatory and bactericidal activity, in addition to promoting tissue repair properties. Nowadays, the overuse of antibiotics in the treatment of infectious diseases, and the appearance of multidrug-resistant bacterial strains, has driven research towards the study of new resistance-
\end{abstract}

Correspondence: Andrea Attilio Basile, Sunstone Science, Via Andrea Solari 8, 20144 Milan, Italy.

Tel.: +39.347.7852322.

E-mail: andreaattilio.basile@fastwebnet.it

Acknowledgments: the authors would like to thank the Italian Society for Oxygen-Ozone Therapy (SIOOT) for its helpful support in the drafting of the study.

Key words: Ozone; Wound healing; Anti-inflammatory therapy; Anterior segment disease.

Contributions: $\mathrm{AB}$ cured case 1 (vernal conjunctivitis), $\mathrm{MC}$ cured case 2 (granulomatous conjunctivitis), GM cured case 3 (persistent dystrophic corneal ulcer), GF prepared the introduction and the references.

Conflict of interest: the authors declare no potential conflict of interest.

Funding: none.

Received for publication: 20 June 2019.

Accepted for publication: 5 July 2019.

${ }^{\circ}$ Copyright: the Author(s), 2019

Licensee PAGEPress, Italy

Ozone Therapy 2019; 4:8377

doi:10.4081/ozone.2019.8377

This article is distributed under the terms of the Creative Commons Attribution Noncommercial License (by-nc 4.0) which permits any noncommercial use, distribution, and reproduction in any medium, provided the original author(s) and source are credited. free antimicrobial agents. In our experience, this new liposomal ozonated oil formulation was used to promote wound healing and treat some inflammatory and infectious pathologies of the anterior segment, such as vernal conjunctivities, granulomatous conjunctivities, persistent dystrophic corneal ulcer, i.e. diseases necessitating adequate anti-inflammatory and regenerative therapy. In these conditions, a topical dosage of one or two drops of collyrium every $4 \mathrm{~h}$ over 3-7 days is generally recommended, even though multiple and frequent instillations are often required to achieve clinical resolution. The use of ozone-based eye drops gave a faster resolution of the inflammatory and/or infectious process, healing of the wounded area, improvement of corneal deepithelization and resolution of the symptomatology (itching, soreness) and clinical sign. In conclusion, from our preliminary results, ozone-based eye drops represent a valid and suitable alternative therapy for the management of external ocular pathologies, both inflammatory and/or infective and traumatic.

\section{Introduction}

Ozone $\left(\mathrm{O}_{3}\right)$ gas, thanks to its great oxidative power, has been widely recognized as one of the best bactericidal, antiviral, and antifungal agents, ${ }^{1,2}$ furthermore, ozone has been used as a clinical therapeutic agent for chronic wounds, such as trophic ulcers, ischemic ulcers, and diabetic wounds. The beneficial effects of ozone on wound healing might be assumed to be due to the upregulation of platelet-derived growth factor (PDGF), transforming growth factor- $\beta$ (TGF- $\beta$ ), the reduction in bacterial load, improvement of the impaired dermal wound healing, or increase in the oxygen tension by ozone exposure in the wound area. ${ }^{2,3}$ Ozone in the gaseous state is extremely reactive and not always suitable as a topical treatment. In saline solution, its concentration reduces quickly with a first-order kinetics and its half-life is $2 \mathrm{~h}$ : this means that in about $24 \mathrm{~h}$ very little ozone will be left in the solution. Interestingly, in spite of its instability, the ozone molecule can be stabilized - for topical use - as an ozonide, an organic ana$\log$ of ozone, formed by the reaction of ozone with an unsaturated compound, such as oleic acid., ${ }^{4}$ Ozonated oil is currently used topically for the treatment of wounds, anaerobic infections, herpetic infections (HSV I and II), trophic ulcers and burns, cellulitis, abscesses, anal fissures, decubitus ulcers (bed sores), fistulae, fungal diseases, furunculosis, gingivitis, and vulvovaginitis. ${ }^{6}$ The use of ozone in certain eye anterior segment pathologies could be providential due to its anti-inflammatory and bactericidal activity, in addition to promoting tissue repair properties. Unfortunately, ozonated oil, as such, is highly irritant for corneal tissue; thus, a specific formulation has been recently developed for ophthalmic use, based on liposomal sunflower ozonated oil plus hypromellose 
(Ozodrop $^{\circledR}$, FB Vision, Ascoli Piceno, Italy), which is extremely biocompatible with the delicate ocular surface tissue.

Ozonated oils have the same properties of gaseous ozone, are well tolerated by biological tissues (corneal tolerability is increased in a liposomal formulation), and their biological activities are related to oxygenated compounds. They are able to eliminate the pathogens by direct oxidation mediated by hydrogen peroxide, lipoperoxyde, and selective cytotoxicity on fast-dividing cells. This occurs through bacterial lysis and cell death, negative regulation on mitochondrial activity in bacteria, and disturbance of viral lithic enzymes, in superimposable manners compared to those of phagocytic cells of the immune system. Moreover, ozone allows a physiological wound healing, minimizing the risk of keloidal scar and also the risk of haze in the cornea. In addition, ozone promotes cell adaptation to oxidative stress and attenuates pathophysiological events mediated by reactive oxygen species. Ozone oxidative preconditioning significantly decreased malondialdehyde levels and increased the activity of superoxide dismutase, glutathione peroxidase, and catalase. Serum interleukin- $1 \beta$ levels tend to decrease with ozone oxidative preconditioning.

In our experience, this new liposomal ozonated oil formulation, specifically intended for ophthalmic use, was used to promote wound healing and treat some inflammatory pathologies in common eye disorders: inflammatory anterior segment diseases necessitating adequate anti-inflammatory therapy, such as conjunctivitis, keratitis, keratoconjunctivitis sicca, and corneal ulcers. In these conditions, a topical dosage of one or two drops of collyrium every 4 h over 3-7 days is generally recommended, even though multiple and frequent instillations are often required to achieve clinical resolution, and some patients tend to become noncompliant with frequent medications. ${ }^{7,8}$ In particular, there is a need for new products for the treatment of ocular pain and inflammation, such as during external ocular infections and inflammations, due to the related risk of blindness. The overuse of antibiotics in the treatment of infectious diseases, and the appearance of multidrug-resistant bacterial strains, has driven research towards the study of antimicrobial agents from essential oils. ${ }^{9}$

Based on the many features and possible applications of ozone, the aim of the present study was a preliminary evaluation of repair and regeneration effect of ozonated oil in liposomes plus hypromellose (Ozodrop ${ }^{\circledR}$ ) instilled 3-4 times a day in external ocular spontaneous pathologies in humans.

\section{Case Reports}

\section{Case \#1}

\section{Vernal conjunctivitis}

A 11-year-old male, suffering from Vernal keratoconjunctivitis (VKC) for years, came to our observation (t0) already receiving the following treatment for a week: deltacortene $12.5 \mathrm{mg}$ per os, topical ophthalmic combination based on betamethasone and chloramphenicol (1 drop t.i.d.) and artificial tears. He reported cyclosporine intolerance. The examination showed conjunctival hyperaemia, papillary conjunctivitis and limbal nodules, corneal nubecole following corneal ulcers.

Without correction, the right eye has a reduced visual acuity of $7 / 10$, and the left eye has a reduced visual acuity of 7-8/10. He presented also lacrimation, thick and filamentous mucous secretion, itching, burning, foreign body sensation, photophobia.

In our first observation ( $\mathrm{t} 0$ ), we add Ozodrop ${ }^{\circledR} 1$ drop (3 times a day) and tear substitutes ( 3 times a day); in a week, oral corticos- teroid was progressively suspended, and the topical steroid and antibiotic therapy was changed with dexamethasone sodium phosphate $0.15 \%$ with taper schedule of 1 drop three times a day, 1 drop two times a day for 10 days and then once a day for 10 days and finally it was suspended.

The patient was monitored after 7 days ( $\mathrm{t} 1)$ after 1-month ( $\mathrm{t} 2)$ and after 2 months ( $\mathrm{t} 3$ ). Already at $\mathrm{t} 1$, and especially at $\mathrm{t} 2 \mathrm{-t} 3$, there was a progressive improvement in symptoms and a reduction, until the disappearance ( $\mathrm{t} 2-3)$, of conjunctival hyperaemia, conjunctival papillae and mucosal secretion, with improvement of visual acuity of the right eye 8-9/10 and of the left eye 9/10 (Figure 1).

\section{Case \#2}

\section{Granulomatous conjunctivitis}

A 19-year-old female patient presented herself to our observation with a unilateral red eye roundish conjunctival nodule, detected by 1 month, inflamed and gradually increasing in size. The clinical features were: conjunctival hyperaemia, watering, itching, soreness at the lesion.

Patient's medical history reveals asthenia for many years, episodes of urticaria of unknown origin, very slight positivity to Borrelia antibodies at the blood tests; presence of anti-thyroid antibodies (Hashimoto) not associated with sarcoidosis, and a
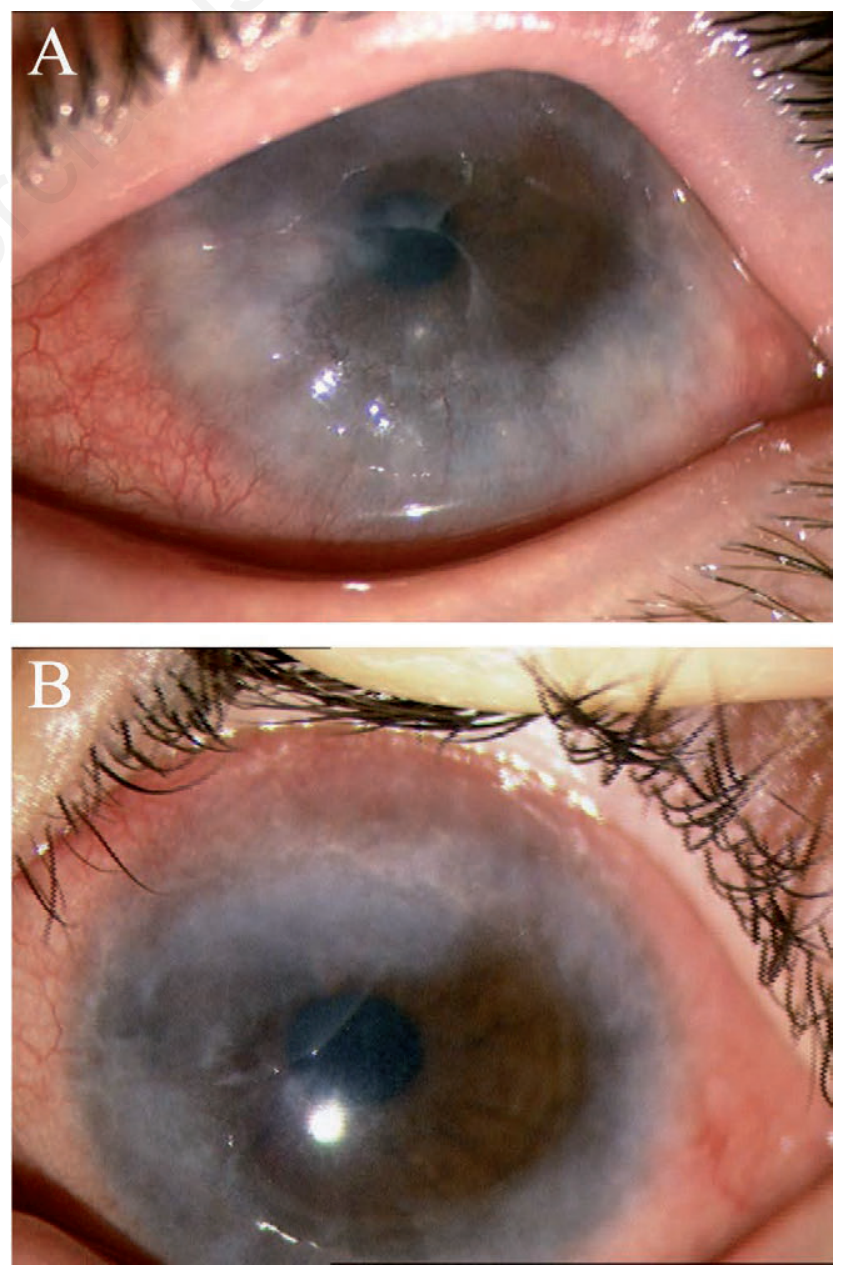

Figure 1. A) The affected eye before starting Ozodrop ${ }^{\circledR}$ treatment. B) The eye after 1 month add-on treatment with Ozodrop ${ }^{\circledR}(t 2)$. 
conjunctival granuloma of unknown origin in immune dysregulation.

A 1-week topical aminoglycosides, topical fluoroquinolones and systemic antibiotic therapy (Amoxicillin) had been prescribed by the attending physician, but the therapeutic regimen didn't solve the symptoms and signs.

We continue the therapy and, considering the complicated clinical situation, associated a topic therapy with ozone-based eye drops in liposomes $\left(\right.$ Ozodrop $\left.^{\circledR}\right), 1$ drop 3 times a day.

After 1 month therapy (t1), at the control visit, we observed that the lesion was almost completely healed and the symptomatology (itching, soreness) and clinical sign (watering) was no longer present (Figure 2).

\section{Case \#3}

\section{Persistent dystrophic corneal ulcer}

A 60 -year-old man came to our observation ( $\mathrm{t} 0$ ) after 6 months of persistent dystrophic corneal ulcer with diffuse epithelial suffering. He was affected by dry eye disease and he was in therapy with antineoplastic drugs (cycles of chemotherapy after surgery) for an intestinal carcinoma. Decrease in ocular symptomatology for corneal hypoesthesia treated repeatedly with re-epithelizing tear substitutes and topical corticosteroids-antibiotics association (betamethasone + chloramphenicol). A fluorescein strip containing
$10 \%$ fluorescein was applied topically to the affected area and was examined with a cobalt blue filter, revealing a temporal lesion. We didn't observe any changes in color and surface of the fluorescence area, indicating absence of corneal leakage.

A 14-day regimen of topic Ozodrop ${ }^{\circledR}, 3$ times a day, was prescribed in add-on at the starting therapy. At the control visit, after 1 week (t1), the patient showed that corneal ulcer was more circumscribed, with improvement of corneal deepithelization (Figure 3).

\section{Discussion}

VKC is a relatively rare, chronic form of ocular allergy that can potentially cause severe visual complications. Affecting mainly children and young adults, it is an IgE- and T cell-mediated disease, leading to a chronic inflammation in which eosinophil, lymphocyte and structural cell activation are involved. Increased levels of inflammatory cytokines have also been demonstrated Conjunctival papillae formation is related to fibroblast activation and production, whereas limbal conjunctival nodules are related to infiltration of inflammatory cells. ${ }^{10}$

Treatment of VKC requires a multiple approach that includes conservative measures and pharmacologic treatment. Patients and
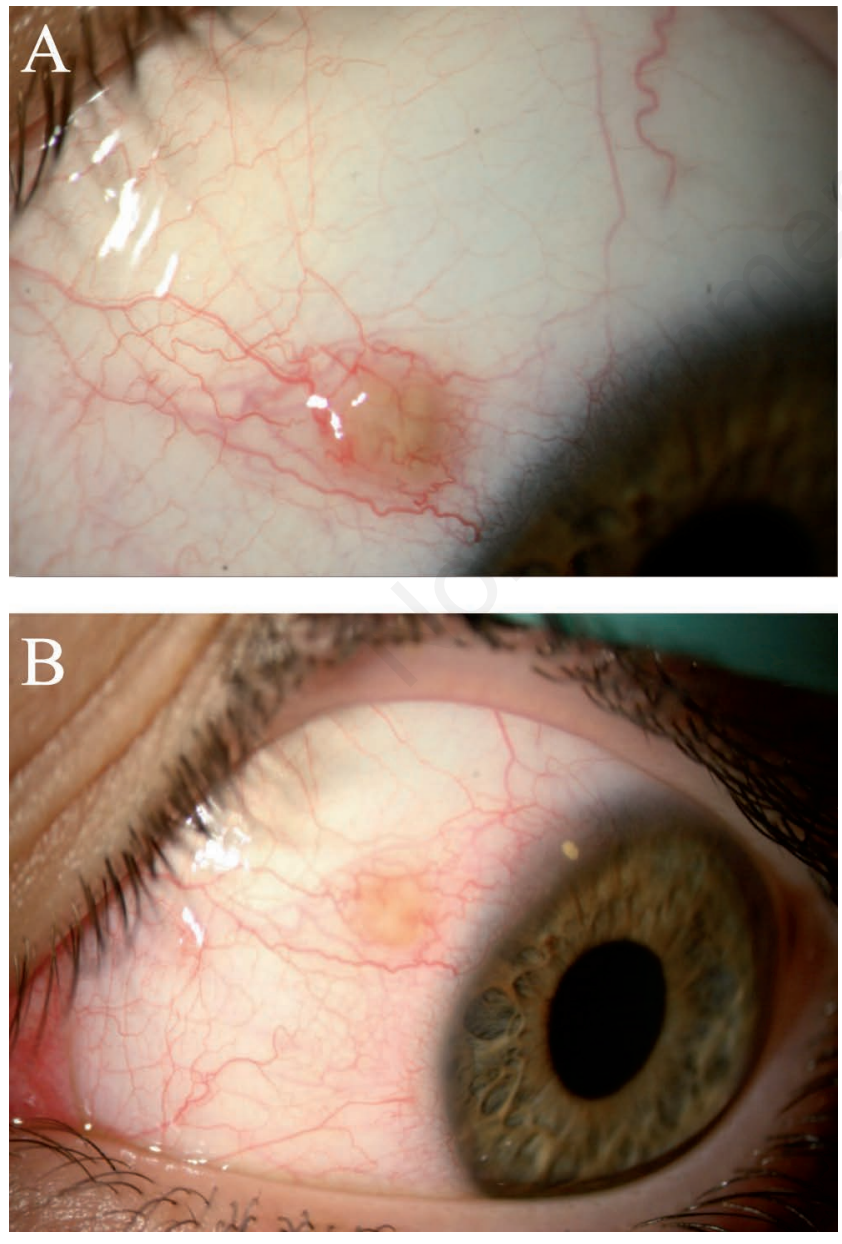

Figure 2. A) The roundish conjunctival nodule before treatment (t0). B) The same nodule after 1 month add-on therapy with Ozodrop $^{\circledR}(\mathbf{t} 1)$.
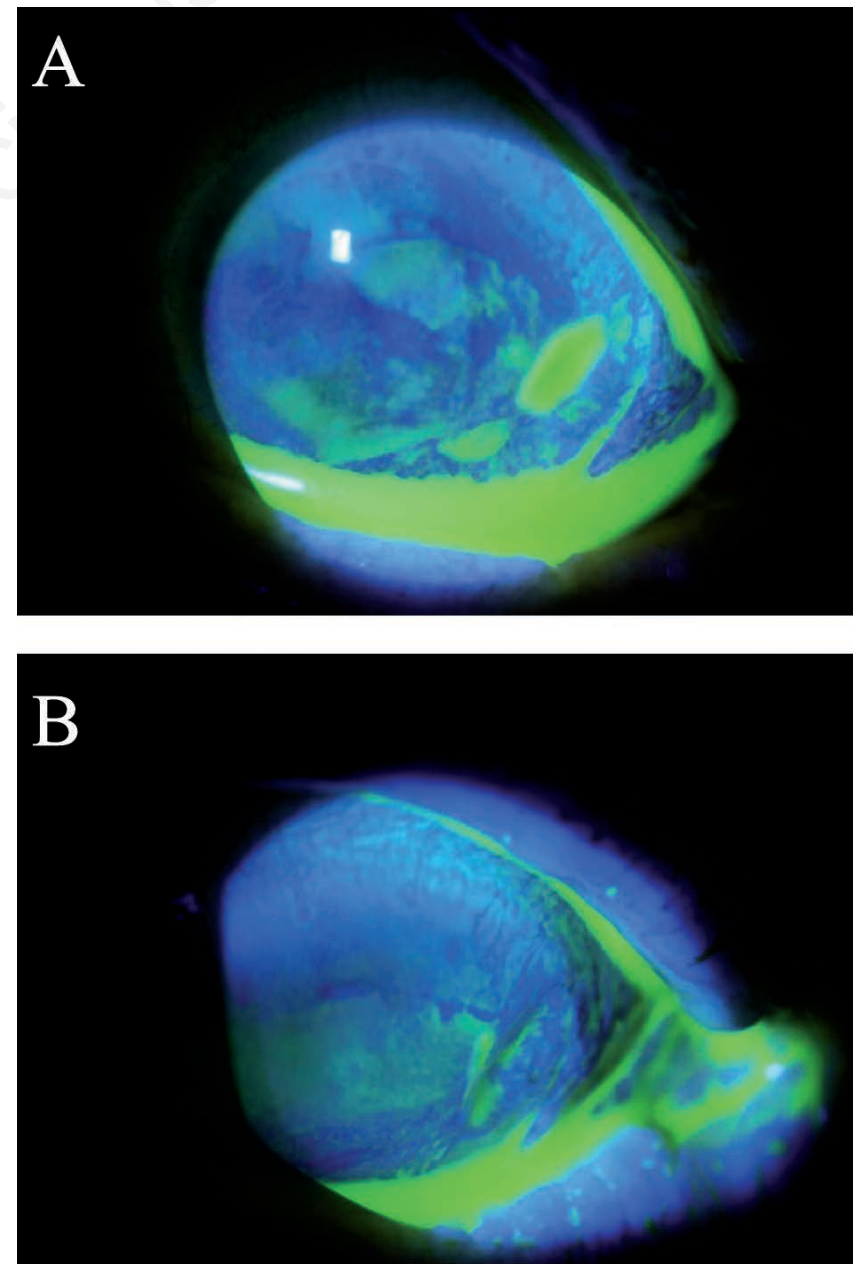

Figure 3. A) Pre-treatment (t0). B) Corneal ulcer after 1 week of Ozodrop ${ }^{\circledR}$ therapy (t1). 
parents should be made aware of the long duration of disease, its chronic evolution and possible complications. Treatment should be based on the duration and frequency of symptoms and the severity of corneal involvement. Mast cell stabilizers and antihistamines have been proven to be effective for the treatment of mild to moderate forms of VKC. In the most severe cases, topical steroids can be used as rescue medication to reduce conjunctival and corneal inflammation.

Ozone can improve the inflammation response pathways (reduction of inflammatory cytokines), the modulation of tissue repair (PDGF, TGF- $\beta$ ) with improvement of corneal transparency; furthermore, ozone can help the progressive adaptation to oxidative stress and increase anti-oxidant systems (Superoxide dismutase, Glutathione reductase, Catalase).

Ozone can have a role in the immune regulation through balance between T-helper 1 (Th1) and T-helper 2 (Th2) activity (reduction of the TH2 overexpression - typical of the vernal conjunctivitis and facilitated by the continuous use of cortisonics); furthermore, it could preserve the ocular surface integrity eliminating the epitheliotossic effect of the antibiotic-therapy thanks to the bactericide characteristics of the ozone.

Though less common than papillary or follicular conjunctivitis, granulomatous conjunctivitis does occur. Clinically, the nodular elevations observed in granulomatous conjunctivitis may be difficult to distinguish from follicles, but clinical history and other systemic symptoms may point to the diagnosis. Granulomatous conjunctivitis in association with preauricular lymphadenopathy is known as Parinaud oculoglandular syndrome. Bacteria such as Bartonella henselae (cat-scratch disease) and Francisella tularensis (tularemia), mycobacteria (e.g., tuberculosis), and treponemes (e.g., syphilis) are possible causes. The diagnosis can be made by serology, culture, polymerase chain reaction, or a combination of these. There are also non-infectious causes of granulomatous conjunctivitis. Sarcoidosis may involve all ocular tissues, including the conjunctiva. It manifests as small, tan nodules, primarily within the forniceal conjunctiva. The nodules are often present even in the absence of an obvious conjunctivitis, that is, in noninjected, asymptomatic eyes. Treatment varies by etiology.

Through the treatment with topical application of ozonized oils in liposome solution, it's possible to obtain the reduction of the infections and the inflammation, proved by the reduction of IL-1. Ozone has a wide-IL-1 is the first cause of the physiological inflammation and in the case of its persistence, in the chronobiology of the inflammatory process, which is a signal, together with the IL-6, of chronicization.

Ozone destroys bacteria by attacking the glycoproteins and glycolipids in the cell membrane results in rupture of the cell. Moreover, $\mathrm{O}_{3}$ attacks the sulfhydryl groups of certain enzymes results in disruption of normal cellular enzymatic activity and loss of function. Ozone also attacks the purine and pyrimidine bases of nucleic acids which results in damage to DNA. The antimicrobial capacity of Ozone includes not only bacteria, but molds, viruses, and protozoa. ${ }^{11}$ Ozone treatment could be a useful support in granulomatous conjunctivitis thanks to its antiseptic broad-spectrum and its anti-inflammatory activity.

A corneal ulcer is a loss of corneal tissue, often associated with inflammation. Most corneal ulcers are infectious, including bacterial and viral etiologies. non-infectious ulcers may be due to chem- ical burns or autoimmune, toxic, neurotrophic, or other causes, e.g. the use of some systemic drugs.

The beneficial effect of ozonized oils in liposome in these conditions exploits the increase of the growth factors, normally deficient in case of delay in reepithelialisation and low tissue repair. After the instillation of the topical ocular ozonized oils, it is observed an upregulation of PDGF and TGF- $\beta$, as well as the bactericidal and anti-inflammatory action already described.

\section{Conclusions}

This preliminary in vivo study demonstrated that ozone-based eye drops have an anti-inflammatory activity, in addition to promoting tissue repair and an antibacterial activity. All of these beneficial and therapeutic effects are contained in a unique ocular preparation, reducing the risk of non-cooperation of patients in receiving medications. Ozone-based eye drops are very attractive due to their versatility and to various potential uses in ophthalmology. In conclusion, from our preliminary results, ozone-based eye drops represent a valid and suitable alternative therapy for the management of external ocular pathologies, both inflammatory and/or infective and traumatic.

\section{References}

1. Elvis AM, Ekta JS. Ozone therapy: a clinical review. J Nat Sci Biol Med 2011;2:66-70.

2. Kim HS, Noh SU, Han YW, et al. Therapeutic effects of topical application of ozone on acute cutaneous wound healing. J Korean Med Sci 2009;24:368-74.

3. Bocci VA, Zanardi I, Travagli V. Ozone acting on human blood yields a hormetic dose-response relationship. J Transl Med 2011;9:66.

4. Valacchi G, Fortino V, Bocci V. The dual action of ozone on the skin. Br J Dermatol 2005;153:1096-100.

5. Bocci V. Oxygen-ozone therapy: a critical evaluation. Dordrecht: Kluwer Academic Publisher; 2002.

6. Bocci V. Ozone: a new medical drug. Dordrecht: Springer; 2005.

7. Hendrix DV. Diseases and surgery of the canine conjunctiva and nictitating membrane. Hoboken: Blackwell Publishing; 2007.

8. Baeyens V, Felt-Baeyens O, Rougier S, et al. Clinical evaluation of bioadhesive ophthalmic drug inserts (BODI) for the treatment of external ocular infections in dogs. J Control Release 2002;85:163-8.

9. Sechi LA, Lezcano I, Nunez N, et al. Antibacterial activity of ozonized sunflower oil (Oleozon). J Appl Microbiol 2001;90: 279-84.

10. Addis H, Jeng BH. Vernal keratoconjunctivitis. Clin Ophthalmol 2018;12:119-23.

11. Megahed A, Aldridge B, Lowe J. The microbial killing capacity of aqueous and gaseous ozone on different surface contaminated with dairy cattle manure. PLoS One 2018;13:e0196555. 